\title{
A Unique Presentation of Bone Marrow Involvement in Gastrointestinal Tract Carcinomas: A Case Series
}

\author{
A Sekaran ${ }^{1}$, M Chandolu $^{1}$, B Patodiya ${ }^{2}$, A Patil $^{1}$, DN Reddy ${ }^{2}$ \\ Department of Pathology ${ }^{1}$ and Gastroenterology ${ }^{2}$, AIG Hospitals, Hyderabad, Telangana, India.
}

\section{Corresponding Author:}

Dr Anuradha Sekaran

Email: dr.sanuradha@aighospitals.com

This is an Open Access article distributed under the terms of the Creative Commons Attribution License (creativecommons.org/ licenses/by/3.0).

Received Accepted Published
December 11, 2020

February 23, 2021

May 10, 2021

\begin{abstract}
Background: Metastasis from gastrointestinal tract cancer is usually seen in the liver, peritoneum and lymph nodes and less frequently in ovaries, lungs, central nervous system and bone. Bone marrow is a rare region for metastasis and its involvement is associated with worst prognosis. Case Report: We hereby report cases of gastric carcinoma (poorly differentiated adenocarcinoma, diffuse type) and caecal adenocarcinoma metastasizing to the bone marrow. Conclusion: Awareness about unusual sites of metastasis is necessary since these cases are associated with poor prognosis.
\end{abstract}

Keywords: Adenocarcinoma, Bone Marrow, Cecal Neoplasms, Liver, Lung.

\section{Introduction}

Disseminated carcinomatosis of the bone marrow is characterized by widespread bone metastasis from solid tumors with haematological manifestation. This disease is frequently occurs with gastric or colonic cancer among solid tumors although its incidence is very rare. The gastric cancer is the third leading cause of cancer deaths worldwide with fifth highest incidence among cancers [1] while colorectal cancer (CRC) is the fourth most incident cancer and third most commonly diagnosed form of cancer globally [2]. Metastasis in gastric and colorectal cancer is usually in the liver, peritoneum and lymph nodes and less frequently in ovaries, lungs, central nervous system and bone. Bone marrow is a rare region for metastasis and its involvement is associated with worst prognosis [3]. Development of metastasis is a concern for patients and clinicians because it is fatal and causes mass-effect and meddles with homeostasis. During the planning of treatment, it is important to know the nodal status and metastasis of the neoplasm for definitive treatment. Marrow involvement in solid malignancy is a strong prognostic factor and strongly influence the tolerance to chemotherapy owing to myelotoxicity. We report cases of gastric carcinoma (poorly differentiated adenocarcinoma, diffuse type) and caecal adenocarcinoma metastasizing to the bone marrow.

\section{Case Reports}

\section{Case 1}

A 40-year-old female with a past history of trauma to the back presented with pain in the sacrum and lumbar region. The MRI lumbar spine revealed mild diffuse altered marrow signal intensity, sacralization of L5 vertebra and L3-L4, L4-L5 disc bulges and disc prolapse. Her complete blood picture showed severe anemia with thrombocytopenia. The bone marrow aspiration and biopsy done in view of anemia and thrombocytopenia revealed metastatic deposits of adenocarcinoma. Patient also had epigastric pain for which endoscopy was done, which revealed a small clean base ulcer in greater curvature of stomach, colonoscopy revealed multiple ulcers in ileocecal valve and aphthous ulcers in terminal ileum. Multiple biopsies were taken from the ulcerated areas. The gastric biopsy 
revealed a poorly differentiated adenocarcinomadiffuse type with few signet ring cells. The ulcers in ileocecal valve and terminal ileum showed moderate active inflammation. The patient happened to have ovarian metastasis as well. As a treatment to this disseminated malignancy, 10 cycles of FOLFOX (Folinic acid, fluorouracil, and oxaliplatin) were given but unfortunately patient succumbed to death.

\section{Case 2}

A63-year-old male presented with a chief complaint of pain in the lower back radiating to both the legs. He also had complained of chest pain, loss of appetite and weight loss of $20 \mathrm{~kg}$ in last 3 months. His complete blood picture showed neutrophilic leucocytosis. The CT scan of spine showed multiple tiny lucent and sclerotic areas in multiple bones. PET-CT was done in view of suspected malignancy which showed heterogeneous increase in FDG uptake in the entire skeleton with mild FDG uptake in the region of cardia and body of stomach. The gastric cardia also showed mild mural thickening on CT scan. Bone marrow aspiration and biopsy revealed metastatic deposits from adenocarcinoma. Upper gastrointestinal endoscopy showed edematous and nodular mucosa in the cardia and proximal body of stomach. Biopsy done from the stomach lesion revealed moderately to poorly differentiated adenocarcinoma.

\section{Case 3}

A 50-year-old male presented with a chief complaint of abdominal pain associated with vomiting. His complete blood picture revealed pancytopenia. Bone marrow aspiration and biopsy were done in view of pancytopenia, which on histology showed atypical cells suggestive of metastatic deposits of adenocarcinoma. Endoscopic-ultrasoundguided fine needle biopsy (EUS-FNB) from paraaortic lymph node showed metastatic deposits of adenocarcinoma. Triphasic CT showed mild thickening of ileo-cecal junction, enlarged para

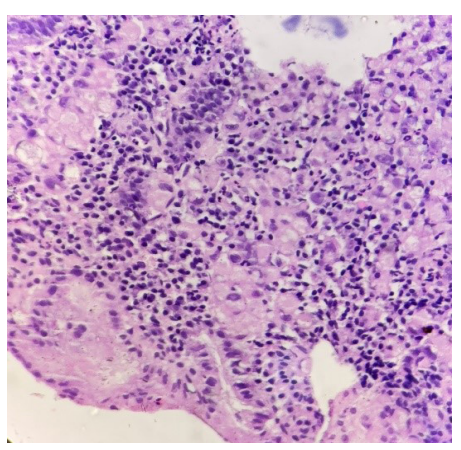

Fig.1: Gastric biopsy showing poorly differentiated adenocarcinoma with signet ring cells [HE, 10×].

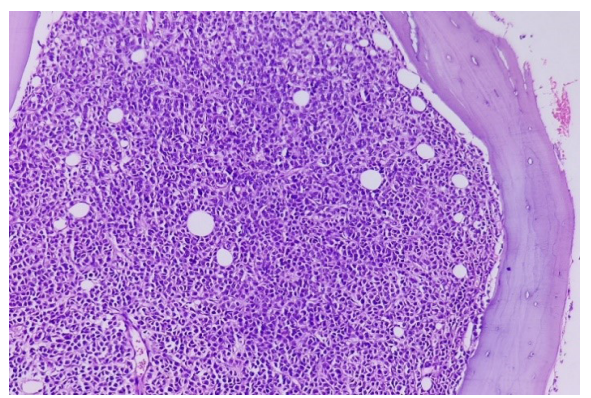

Fig.2: Bone marrow biopsy showing metastatic deposits from gastric adenocarcinoma [HE, $10 \times$ ].

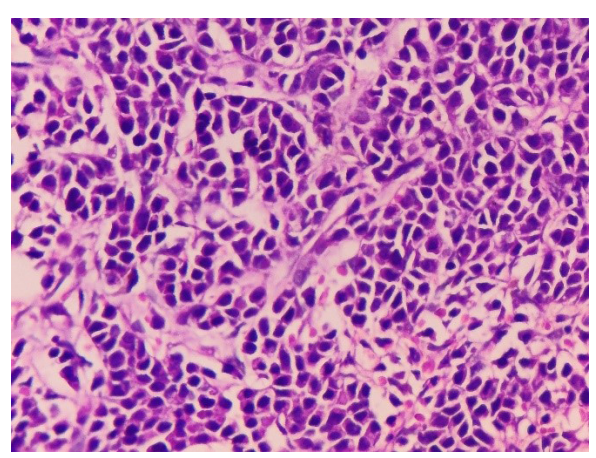

Fig.3: Gastric biopsy showing poorly differentiated adenocarcinoma [HE, 40×].

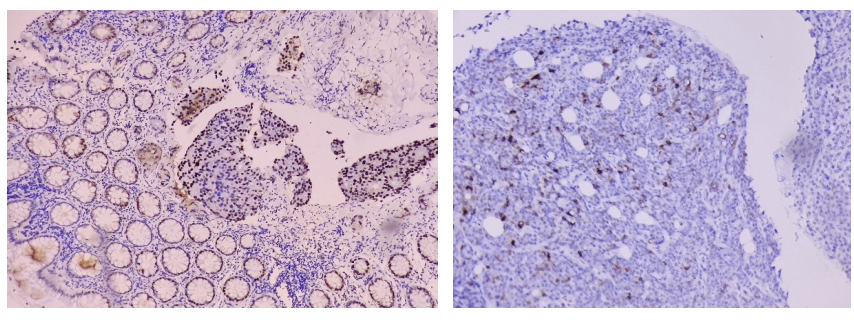

Fig.4: Pan CK showing cytoplasmic positivity in the tumour cells in bone marrow biopsy [PanCk 10×]/ MUC5AC showing cytoplasmin positivity in tumor cells [10 $\times]$. 
celiac, hepatoduodenal, para-aortic, aorto caval, ileo colic lymph nodes with few showing matting and minimal pleural effusion and ascites. PET-CT showed diffuse uptake in the bone marrow and ileocecal region. Colonoscopy showed edematous mucosa with nodularity in the cecum and narrowing of ileo-caecal valve. Cecal and ileo-caecal valve biopsies on histopathological examination showed features of adenocarcinoma.

\section{Discussion}

The usual sites of metastasis from a gastrointestinal carcinoma are liver, peritoneum and lymph nodes. Bony metastasis are common from breast carcinoma, prostatic carcinoma and pancreatic carcinoma. Bony metastasis from GI cancers are relatively rare and bone marrow metastasis is an even rarer event which is associated with worst prognosis. The most common hematologic abnormality in patients with bone marrow metastasis in gastric cancer is thrombocytopenia [3]. The metastatic path of spread in gastric carcinoma is generally hematogenous and also via lymphatics [4]. The mechanism thought for bone marrow metastasis is through the following processes: (i) cancer cells break away from the primary lesion and enter the bloodstream; (ii) these cells survive and multiply in the bone marrow cavity; (iii) the differentiation and activation of osteoclasts occur; and (iv) the cancer cells proliferate in the bone microenvironment [4]. Kim et al. reported the prevalence of gastric adenocarcinoma with bone marrow metastasis to $0.024 \%$ [5].

The incidence for bone marrow metastasis in gastric cancer patients is low, therefore the clinical features and treatment options for such patients has not been established properly [6]. Nevertheless, the most common cause of death in patients with gastric cancer and bone marrow metastasis was disease progression, not chemotherapy-related hematologic toxicities. It is difficult to determine whether the intensity of cytotoxic chemotherapy should be increased to prolong patient survival

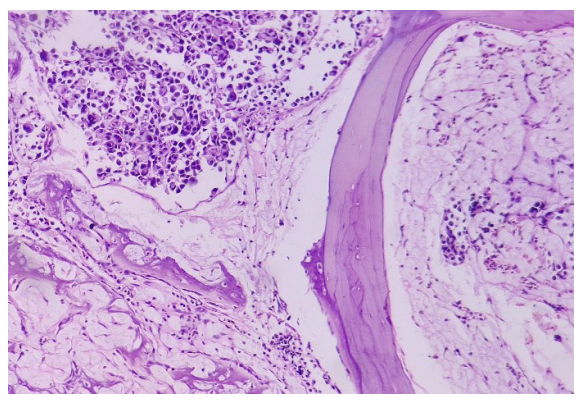

Fig.5: Bone marrow with metastatic deposit of adenocarcinoma with pools of extracellular mucin [Alcian blue-PAS, $10 \times]$.

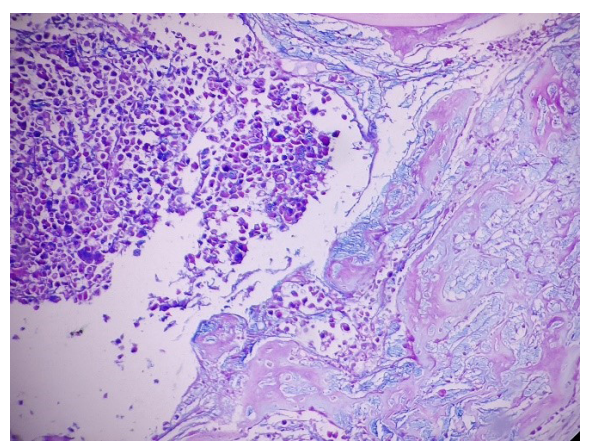

Fig.6: Bone marrow with metastatic deposit of caecal adenocarcinoma [HE, 10×].

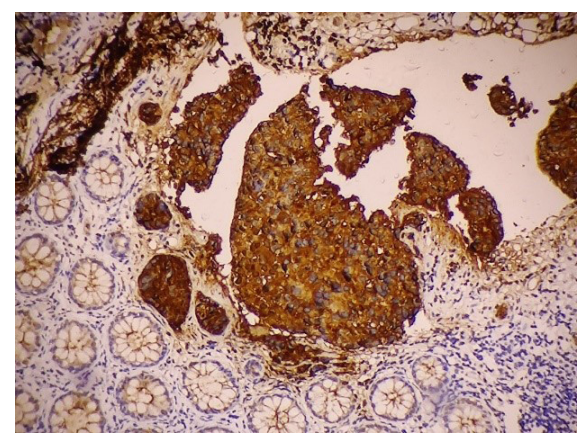

Fig.7: CDX2.2 showing nuclear positivity and MCEA showing cytoplasmic positivity in colonic tumour [20×].

or lowered to avoid toxicity. Thrombocytopenia (81\%) was the most common peripheral blood finding. In case of metastasis to the bone marrow, the cancer cells proliferate diffusely in the marrow space and this leads to disseminated carcinomatosis which induces bone destruction and haematological complications such as disseminated intravascular coagulopathy (DIC), bi/pancytopenia or profound 
myelosuppression after chemotherapy [4]. At the time of diagnosis with gastrointestinal tract adenocarcinoma, clinicians should suspect bone marrow metastasis and consider a bone marrow biopsy if patients present with prominent thrombocytopenia [7].

The importance of recognising marrow involvement is mainly prognostic but also prediction of toxicity of chemotherapy. The most common histological types associated with bone marrow metastasis are poorly differentiated adenocarcinoma and signet ring cell carcinoma [8]. The tolerance of chemotherapy is poor in marrow involved cases and patient by default receive lower dose than non-marrow involved cases. In case of metastatic disease, initiation of chemotherapy starts at a lower dose for the $1^{\text {st }}$ cycle of chemotherapy and then the dose is escalated based on tolerance. This is in contrast with non-metastatic cases where treatment is initiated at full dose and then de-escalated based on toxicity. Hence distant metastasis, especially bone marrow metastasis is particularly of clinical importance for medical oncologists in planning the treatment. In both gastric and colon cancer the most accepted chemotherapy protocol of choice contains capecitabine or 5-fluorouracil (5-FU). Theoretically, 5-FU proved to be more toxic as dose titration is not possible once the chemotherapy is infused, while with capecitabine dose titration is possible based on the $10^{\text {th }}$ day blood picture. In the experience of one of the co-authors, CAPOX regimen (capecitabineoxaliplatin) is preferred in marrow involved cases. Moreover, two weekly CAPOX regimen is already proven to be equally efficacious but less toxic than three weekly regimens. Use of granulocyte colony stimulating factor (GCSF) as primary prophylaxis in this scenario is beneficial, even though these regimens are not severely myelotoxic. Epirubicinbased protocols are out of vogue for gastric cancer and may prove difficult to tolerate in marrow involved cases. For colon cancer if any targeted therapy like cetuximab is planned it can be added from the second cycle depending on baseline platelet count.

Chemotherapy is the treatment of choice in cases of advanced gastric carcinoma which promises a better quality of life than with symptomatic and supportive alone [9]. Advanced signet ring cell carcinoma carries a poorer prognosis compared to other types of gastric cancer. Mean survival time for bone marrow metastasis from gastric carcinoma is 4-5 months [7]. Viehl et al. [10] in their prospective trial found that $38 \%$ of stage I-III colon cancer patients could have bone marrow micro-metastasis (BMM). They concluded that BMM are independent prognostic factors for both disease-free survival (DFS) and overall survival (OS); however, the clinical significance of BMM is still debatable [10]. Because of the lack of prospective studies of gastro-intestinal tract cancer patients with bone marrow metastasis an optimal chemotherapy regimen is still not known. The prognostic significance of bone marrow metastasis from colon cancer is controversial because the cases reported in the literature are limited and comparison of data is difficult due to somewhat different disease definitions [11].

\section{Conclusion}

This case series highlights some important points related to diagnosis and treatment regimen of gastrointestinal tract carcinoma presenting with bone marrow metastasis. New insights on the chemotherapeutic regimens are introduced which are less myelotoxic and can be used to treat these patients. One must be aware of unusual sites of metastasis since these cases are associated with a worst prognosis with a median survival rate of 4-5 months.

Contributors: AS: Conception of study, manuscript writing, histopathology and clinical revision; MC, AP: Data collection, analysis, manuscript editing; BP, DNR: critical inputs into the manuscript and patient management. AS will act as a study guarantor. All authors approved the final version of this manuscript and are responsible for all aspects of this study. Funding: None; Competing interests: None stated. 


\section{References}

1. Rawla P, Barsouk A. Epidemiology of gastric cancer: global trends, risk factors and prevention. Prz Gastroenterol. 2019;14(1):26-38.

2. Rawla P, Sunkara T, Barsouk A. Epidemiology of colorectal cancer: incidence, mortality, survival, and risk factors. Prz Gastroenterol. 2019;14(2):89-103.

3. Ergun Y, Uncu D, Yazici O, Ucar G, Konca Karabuga E, Zengin N. Gastric cancer patients with bone marrow metastasis: A single-center experience and review of the literature. EJMO. 2017;1(3):160-163.

4. Iguchi H. Recent aspects for disseminated carcinomatosis of the bone marrow associated with gastric cancer: What has been done for the past, and what will be needed in future? World J Gastroenterol. 2015;21(43):1224912260.

5. Kim HS, Yi SY, Jun HJ, Lee J, Park JO, Park YS, et al. Clinical outcome of gastric cancer patients with bone marrow metastases. Oncology. 2007;73:192-197.

6. Ekinci AŞ, Bal O, Ozatlı T, Türker I, Eşbah O, Demirci A, et al. Gastric carcinoma with bone marrow metastasis: a case series. J Gastric Cancer. 2014;14(1):54-57.
7. Kwon JY, Yun J, Kim HJ, Kim KH, Kim SH, Lee SC, et al. Clinical outcome of gastric cancer patients with bone marrow metastases. Cancer Res Treat. 2011;43(4):244-249.

8. Dittus C, Mathew H, Malek A, Negroiu A. Bone marrow infiltration as the initial presentation of gastric signet ring cell adenocarcinoma. J Gastrointest Oncol. 2014;5(6):E113-E116.

9. Orditura M, Galizia G, Sforza V, Gambardella V, Fabozzi A, Laterza MM, et al. Treatment of gastric cancer. World J Gastroenterol. 2014;20(7):1635-1649.

10. Viehl CT, Weixler B, Guller U, Dell-Kuster S, Rosenthal $\mathrm{R}$, Ramser $\mathrm{M}$, et al. Presence of bone marrow micrometastases in stage I-III colon cancer patients is associated with worse disease -free and overall survival. Cancer Med. 2017;6(5):918-927.

11. Assi R, Mukherji D, Haydar A, Saroufim M, Temraz S, Shamseddine A. Metastatic colorectal cancer presenting with bone marrow metastasis: a case series and review of literature. J Gastrointest Oncol. 2016;7(2):284-297. 